Pacific Journal of Mathematics

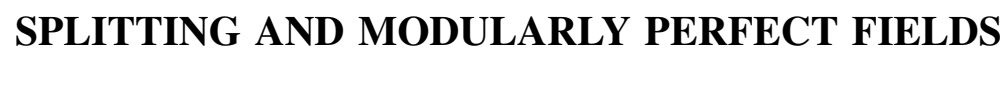




\title{
SPLITTING AND MODULARLY PERFECT FIELDS
}

\author{
James K. Deveney and John N. Mordeson
}

Let $K$ be a field of characteristic $p \neq 0$. A field extension $L / K$ is said to split when there exist intermediate fields $J$ and $D$ of $L / K$ where $J$ is purely inseparable over $K, D$ is separable over $K$ and $L=J \otimes_{K} D . \quad K$ is modularly perfect if $\left[K: K^{p}\right] \leqq p$. Every finitely generated extension of a modularly perfect field splits. This paper develops criteria for an arbitrary extension $L / K$ to split and presents an example of an extension of a modularly perfect field which does not split. Necessary and/or sufficient conditions are also developed for the following to hold for an extension $L / K$ : (a) $L^{\prime} / K$ splits for every intermediate field $L^{\prime}$; (b) $L^{\prime} / K$ is modular for every intermediate field $L^{\prime}$; (c) $L / L^{\prime}$ splits for every intermediate field $L^{\prime}$; (d) $L / L^{\prime}$ is modular for every intermediate field $L^{\prime}$.

Introduction. Let $K$ be a field of characteristic $p \neq 0$. A field extension $L / K$ is said to split when there exists intermediate fields $J$ and $D$ of $L / K$ where $J / K$ is purely inseparable, $D / K$ is separable, and $L=J \otimes_{K} D$. It is a classic result that any normal algebraic field extension $L / K$ must split. Recent papers have been concerned with nonalgebraic extensions $L / K$. Suppose there exists an intermediate field $J$ of $L / K$ such that $L / J$ is separable and $J / K$ is purely inseparable (hence $J=L \cap K^{p^{-\infty}}$ ). Under certain conditions, namely, if $L$ has a separating transcendence basis over $J$ [4], or if $J$ is of bounded exponent over $K$ [5], then $L / K$ must split. That some conditions must be put on $L / J / K$ is illustrated by an example in [1].

A field extension $L / K$ is called modular if $L^{p^{n}}$ and $K$ are linearly disjoint for all $n$. The importance of modular extensions was first observed by Sweedler [11] who used this property to characterize purely inseparable extensions of bounded exponent which were tensor products of simple extensions. In [4] it was shown that if $L / K$ is an (arbitrary) modular extension then there must exist an intermediate field $J$ such that $L / J$ is separable and $J / K$ is purely inseparable modular. It follows that any finitely generated modular extension must split. In [5], a field $K$ such that $\left[K: K^{p}\right] \leqq p$ is called modularly perfect. Such fields are characterized by the fact that any extension $L$ of such a field $K$ must be modular over $K$. In view of the above results, a natural question is whether every extension of a modularly perfect field $K$ must split. In part I we develop a number of criterion for a field extension to split. We construct an extension $L$ of a 
modularly perfect field $K$ which does not split. The field $L$ also does not have a distinguished separable subfield [3], where $D$ is distinguished in $L / K$ if and only if $D / K$ is separable and $L \cong D\left(K^{p^{-\infty}}\right)$. In the remainder of the paper we determine necessary and/or sufficient conditions for the following to hold for an extension $L / K$ :

(a) $L^{\prime} / K$ splits for every intermediate field $L^{\prime}$;

(b) $L^{\prime} / K$ is modular for every intermediate field $L^{\prime}$;

(c) $L / L^{\prime}$ splits for every intermediate field $L^{\prime}$;

(d) $L / L^{\prime}$ is modular for every intermediate field $L^{\prime}$.

I. Let $L \supseteqq K$ be fields of characteristic $p \neq 0$. An intermediate field $D$ of $L / K$ is called a distinguished separable intermediate field [3] if $D$ is separable over $K$ and $L \subseteq D \bigotimes_{K} K^{p^{-\infty}}$.

REMARK 1.1. The following conditions are equivalent on $K$.

(1) $L / K$ splits for every finitely generated field extension $L / K$.

(2) $\left[K: K^{p}\right] \leqq p$.

(3) $L / K$ splits for every field extension $L / K$ which has a distinguished separable intermediate field.

Proof. $1 \rightarrow 2$ : Suppose $\left[K: K^{p}\right]>p$. Let $x, y$ be $p$-independent in $K$ and let $z$ be transcendental over $K$. Then $L=K\left(z, z x^{p^{-1}}+y^{p^{-1}}\right)$ is a finitely generated extension of $K$ which does not split.

$2 \rightarrow 3$ : By [5, Theorem 6, p. 1180], $L / K$ is modular and whence splits [8, Corollary, p. 607].

$3 \rightarrow 1$ : If $L / K$ is finitely generated, then $L / K$ has a distinguished separable intermediate field.

In $[5$, Theorem $6, p .1180]$ it was shown that $\left[K: K^{p}\right] \leqq p$ if and only if for every field extension $L / K$ there exists a separable field extension $S$ of $K$ (not necessarily in $L$ ) such that $L \subseteq S \bigotimes_{K}\left(K^{p^{-\infty}} \cap L\right)$. Obviously $S$ can be chosen as an intermediate field of $L / K$ if and only if $L / K$ splits. We now develop criterion for an extension $L / K$ to split and present an example of an extension of a modularly perfect field which does not split.

LEMma 1.2. Let $D$ be an intermediate field of $L / K$ such that $L / D$ is purely inseparable and $D / K$ is separable. Then $D$ is maximal separable if and only if $L^{p} \cap D \subseteq K\left(D^{p}\right)$.

Proof. Assume $D$ is maximal and let $b \in L \backslash D$. If $b^{p} \in D \backslash K\left(D^{p}\right)$, then $D(b) / K$ is separable as follows: Let $G$ be a $p$-basis for $K$. Then $G$ is $p$-independent in $D$. Since $b^{p} \in D \backslash K\left(D^{p}\right), G \cup\left\{b^{p}\right\}$ is $p$-independent in $D$. Hence there does not exist $c \in G$ such that $c \in D^{p}\left(b^{p}, G \backslash\{c\}\right)$. Thus $G$ is $p$-independent in $D(b)$ and $D(b) / K$ is separable. However 
this contradicts the maximality of $D$. Thus $b^{p} \in K\left(D^{p}\right)$ and $L^{p} \cap D \leqq$ $K\left(D^{p}\right)$.

Conversely, assume $L^{p} \cap D \subseteq K\left(D^{p}\right)$. If $L=D$, then $D$ is maximal. Suppose $L \supset D$ and let $b \in L \backslash D$ be such that $b^{p} \in D$. Then $b^{p} \in L^{p} \cap$ $D \subseteq K\left(D^{p}\right)$ so $b \in D \otimes_{K} K^{p^{-1}}$. Thus $D(b) / K$ is not separable and hence $D$ is maximal.

THEOREM 1.3. Suppose $L \supseteqq K^{p^{-\infty}}$ and $L / L^{p^{\infty}}$ has a separating transcendence basis. Then $L / K$ splits.

Proof. Since $L \supseteqq K^{p^{-\infty}}, L^{p^{\infty}} \supseteq K^{p^{-\infty}}$. Let $D$ be a maximal separable extension of $K$ in $L^{p^{\infty}}$. We first show $D^{p^{-1}} \cap L^{p^{\infty}} \cong D\left(K^{p^{-\infty}}\right)$. If $b \in D^{p^{-1}} \cap L^{p^{\infty}}$, then $b^{p} \in K\left(D^{p}\right)$ by the previous lemma. Hence $b \in D\left(K^{p^{-1}}\right) \subset D\left(K^{p^{-\infty}}\right)$, as desired. Now $L^{p^{\infty}} / D$ is purely inseparable, and hence $L^{p^{\infty}} / D\left(K^{p^{-\infty}}\right)$ is also purely inseparable. We prove $L^{p^{\infty}}=$ $D\left(K^{p^{-\infty}}\right)$ by showing that each element $b \in L^{p^{\infty}}$ of exponent one over $D\left(K^{p^{-\infty}}\right)$ is actually in $D\left(K^{p^{-\infty}}\right)$. For such $b, b^{p}=\Sigma d_{i} e_{i}$ where $d_{i} \in D$ and $e_{i} \in K^{p^{-\infty}}$. Hence $b=\Sigma d_{i}^{p^{-1}} e_{i}^{p^{-1}}$ where each $d_{i}^{p^{-1}} \in L^{p^{\infty}}$ is of exponent one over $D$. As noted above, each $d_{i}^{p^{-1}} \in D\left(K^{p^{-\infty}}\right)$ and thus $b \in D\left(K^{p^{-\infty}}\right)$ and $L^{p^{\infty}}=D \otimes_{K} K^{p^{-\infty}}$. Now $L / L^{p^{\infty}}$ has a separating trancendence basis and $L^{p^{\infty}} / D$ is purely inseparable. Hence there exists an intermediate field $D^{*}$ of $L / D$ such that $D^{*} / D$ is separable and $L=$ $D^{*} \bigotimes_{D} L^{p^{\infty}}\left[4\right.$, Proposition 1, p. 2]. Thus $L=D^{*} \bigotimes_{D}\left(D \bigotimes_{K} K^{p^{-\infty}}\right)=$ $D^{*} \otimes_{K} K^{p^{-\infty}}$. Since $D^{*} / D$ and $D / K$ are separable, $D^{*} / K$ is separable.

CoRollary 1.4. (1) If $\left[K: K^{p}\right] \leqq p$, then $L / K$ splits for every field extension $L / K$ such that $L / L^{p^{\infty}}$ has a separating transcendence basis.

(2) Conversely, suppose $K / K^{p^{\infty}}$ has a separating transcendence basis. If $L / K$ splits for every field extension $L / K$ such that $L / L^{p^{\infty}}$ has a separating transcendence basis, then $\left[K: K^{p}\right] \leqq p$.

Proof. (1) If $L \supseteq K^{p^{-\infty}}$, then $L / K$ splits by 1.3 . If $L \nsupseteq K^{p^{-\infty}}$, then $\left(L \cap K^{p^{-\infty}}\right) / K$ has bounded exponent. Since $\left[K: K^{p}\right] \leqq p, L / K$ is modular [5, Theorem 1, p. 1177] and hence splits [5, Theorem 3, p. $1178]$.

(2) Suppose $\left[K: K^{p}\right]>p$. Let $T$ be a separating transcendence basis for $K / K^{p^{\infty}}$. Then $T$ is a $p$-basis for $K$ and $|T|>1$. Let $\{x, y\} \subseteq$ $T$ and set $L=K\left(z, z x^{p^{-1}}+y^{p^{-1}}\right)$ where $z$ is transcendental over $K$. If we show $L / L^{p^{\infty}}$ has a separating transcendence basis, we have a contradiction since $L / K$ does not split. Now $T \backslash\{y\} \cup\left\{z, z x^{p^{-1}}+y^{p^{-1}}\right\}$ is a $p$-basis for $L . \quad L^{p^{\infty}} \subseteq \bigcap_{i=1}^{\infty} K\left(L^{p^{2}}\right)=K$, so $L^{p^{\infty}}=K^{p^{\infty}} . K / L^{p^{\infty}}(T)$ is separable algebraic so $L / L^{p^{\infty}}\left(T \backslash\{y\}, z, z x^{p^{-1}}+y^{p^{-1}}\right)$ is separable algebraic 
since $y \in L^{p^{\infty}}\left(T \backslash\{y\}, z, z x^{p^{-1}}+y^{p^{-1}}\right)$. Thus $L / L^{p^{\infty}}$ has a separating transcendence basis.

Proposition 1.5. If $L / K$ splits for every field extension $L / K$ such that $L / L \cap K^{p^{-\infty}}$ is separable, then $\left[K: K^{p}\right]<\infty$.

Proof. Suppose $\left[K: K^{p}\right]=\infty$ and let $\left\{x_{1}, \cdots, x_{n}, \cdots\right\}$ be a $p$ independent subset of $K$. Let $J=K\left(x_{1}^{p^{-1}}, x_{2}^{p^{-2}}, \cdots\right)$ and $L=J\left(z, z^{p^{-1}}+\right.$ $\left.x_{1}^{p^{-2}}, \cdots, z^{p^{-n}}+x_{1}^{p^{-n-1}}+\cdots+x_{n}^{p^{-n-1}}, \cdots\right)$ where $z$ is transcendental over $J$. Since $L$ is the union of a chain of simple transcendental extensions of $J, L / J$ is separable and $L \cap K^{p^{-\infty}}=J$. The proof that $L / K$ does not split is completely analogous to the proof in [1].

Suppose $K$ is a modularly perfect field, i.e., $\left[K: K^{p}\right] \leqq p$. Then for any field $L$ which contains $K, L$ is separable over $L \cap K^{p^{-\infty}}$. If $L \cap K^{p^{-\infty}} \neq K^{p^{-\infty}}$, then $L / K$ must split. We now present an example where $L \supset K^{p^{-\infty}}$ and yet $L / K$ does not split. This example indicates that the result presented in [5, Theorem 6, p. 1180] is in some sense the best possible.

EXAMPLE 1.6. Let $P$ be a perfect field and let $x, y, w_{0}$ be algebraically independent indeterminates over $P$. Set $K=P(y)$ and $L=$ $K^{p^{-\infty}}\left(x, w_{0}, w_{1}^{p^{-1}}, \cdots, w_{n}^{p^{-n}}, \cdots\right)$ where $w_{1}^{p^{-1}}=x^{p^{-1}}+y^{p^{-3}} w_{0}^{p^{-1}}$ and $w_{n}^{p^{-n}}=$

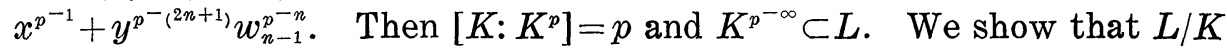
does not split. Assume $L=S \otimes_{K} K^{p^{-\infty}}$ where $S$ is separable over $K$. Consider $S^{\prime}=S\left(x, w_{0}\right)$. Since $S^{\prime}$ is finitely generated over $S, S^{\prime} / K$ has bounded exponent and hence splits since $K$ is modularly perfect [5, Theorem 6, p. 1180]. Let $S^{\prime}=S^{*} \bigotimes_{K} K\left(y^{p^{-t}}\right)$, and hence $L=$ $S^{\prime} \otimes_{K\left(y^{p^{-t}}\right)} K^{p^{-\infty}}$. Now by construction, $K\left(y^{p^{-t}}, x, w_{0}\right) \equiv K_{t} \subseteq S^{\prime}$ and $K_{t} \boldsymbol{\otimes}_{K\left(y^{p^{-t}}\right)} K^{p^{-\infty}}=K^{p^{-\infty}}\left(x, w_{0}\right)$. The fields which lie between $K^{p^{-\infty}}\left(x, w_{0}\right)$ and $L$ are chained and each is a purely inseparable extension of exponent one of the previous one. Hence the same is true for the fields which lie between $K_{t}$ and $S^{\prime}$. Since $K^{p^{-\infty}}\left(x, w_{0}\right) / K_{t}$ is also modular, it follows that $L / K_{t}$ is modular, and in fact $\left[K_{t}^{p^{-n}} \cap\right.$ $\left.L: K_{t}\right]=p^{2 n}$ for all $n$. Since any finitely generated extension of $K_{t}$ in $L$ is contained in $K_{t}^{p^{-n}} \cap L$ for some $n$, and $K_{t}^{p^{-n}} \cap L$ is modular over $K_{t}$ with two elements in any subbase, we conclude that any finitely generated extension of $K_{t}$ in $L$ must be modular over $K_{t}$ [9, Proposition 2.5, p. 76].

We now show that there is a field $M$ which lies between $L$ and $K_{t}$ which is not modular over $K_{t}$. Since $w_{n}^{p^{-n}}=x^{p^{-1}}+y^{p^{-(2 n+1)}} w_{n-1}^{p^{-n}}$, for large $n, w_{n}^{p^{-n}}$ will not be of exponent $n$ over $K_{t}$. Thus assume $w_{n-1}^{p^{-(n-1)}}$ is of exponent $n-1$ over $K_{t}$ and $w_{n}^{p^{-n}}$ is not of exponent $n$. It follows that $\left(y^{p^{-(2 n+1)}}\right)^{p^{n}}=y^{p^{-(n+1)}}$ is not an element of $K_{t}$. Let $M=$ $K_{t}\left(y^{p^{-(2 n+1)}}, w_{n}^{p^{-n}}\right)$. If we show every higher derivation on $M$ over $K_{t}$ 
maps $y^{p^{-(n+1)}}$ to $0, M / K_{t}$ is not modular [11, Theorem 1, p. 403]. Recall that a higher derivation of $M$ over $K_{t}$ is a sequence of $K_{t^{-}}$ linear maps $D^{(s)}=\left\{D_{0}=I, D_{1}, \cdots, D_{s}\right\}$ of $M$ into itself such that $D_{m}(b c)=$ $\sum_{i=0}^{m} D_{i}(b) D_{m-i}(c)$ for all $b, c \in M, m=0, \cdots, s$. We shall need the direct corollary to $\left[13\right.$, p. 436] that $D_{m}\left(b^{p^{r}}\right)=0$ if $p^{r} \nmid m$ and $D_{m}\left(b^{p^{r}}\right)=$ $\left(D_{m / p^{r}}(b)\right)^{p^{r}}$ if $p^{r} \mid m$. We follow the method of Sweedler [11, Example 1.1 , p. 405]. Assume there exists $D_{s}$ such that $D_{s}\left(y^{p^{-(n+1)}}\right) \neq 0$. Then

$$
\begin{aligned}
w_{n-1}\left[D_{s / p^{n}}\left(y^{p-(2 n+1)}\right)\right]^{p^{n}} & =D_{s}\left(w_{n-1} y^{p^{-(n+1)}}\right) \\
& =D_{s}\left(x^{p^{n-1}}+y^{p^{-(n+1)}} w_{n-1}\right) \\
& =\left[D_{s / p^{n}}\left(x^{p^{-1}}+y^{p^{-(2 n+1)}} w_{n-1}^{p^{-n}}\right)\right]^{p^{n}} .
\end{aligned}
$$

Hence

$$
w_{n-1}=\frac{\left[D_{s / p^{n}}\left(x^{p^{-1}}+y^{p^{-(2 n+1)}} w_{n-1}^{p^{-n}}\right]^{p^{n}}\right.}{\left[D_{s / p^{n}}\left(y^{\left.p^{-(2 n}+1\right)}\right)\right]^{p^{n}}}
$$

which is an element of $M^{p^{n}}$.

Thus $w_{n-1}^{p^{-n}} \in M$ and hence $x^{p^{-1}} \in M$, a contradiction since $x^{p^{-1}}$ is not in $L$.

In the previous example, $L$ is of transcendence degree two over $K$. The next result illustrates that this is the least degree possible for an extension which does not split.

Proposition 1.7. $\left[K: K^{p}\right] \leqq p$ if and only if $L / K$ splits for every field extension $L / K$ of transcendence degree one.

Proof. Suppose $\left[K: K^{p}\right] \leqq p$. As usual it suffices to consider the case where $L \supseteqq K^{p^{-\infty}}$. If $L=L^{p}$, then $L=L^{p^{\infty}}$ and $L / K$ splits by 1.4 . If $L \supset L^{p}$, we show $L$ has a separating transcendence basis over $L^{p^{\infty}}$. Since $L \supset K^{p^{-\infty}}, L / L^{p^{\infty}}$ has transcendence degree 1 . Let $B$ be a $p$-basis for $L$. Since $B$ is algebraically independent over $L^{p^{\infty}}, B$ consists of exactly one element and $L / L^{p^{\infty}}(B)$ is algebraic. Since $L / L^{p^{\infty}}(B)$ is separable, $B$ is a separating transcendence basis for $L / L^{p^{\infty}}$ and 1.3 applies.

Conversely, suppose $\left[K: K^{p}\right]>p$. Let $x, y$ be $p$-independent in $K$ and let $z$ be transcendental over $K$. Then $L / K$ does not split where $L=K\left(z, z x^{p^{-1}}+y^{p^{-1}}\right)$.

As Example 1.6 illustrates, not every extension $L / K$ of a modularly perfect field need split. The following result gives several criteria for such an extension to split.

THeOREM 1.8. Assume $\left[K: K^{p}\right]=p$ and $L \supseteqq K^{p^{-\infty}}$. The following are equivalent. 
(1) $L / K$ splits.

(2) There exists a maximal separable extension $D$ of $K$ in $L$ such that $L$ is modular over $D$.

(3) There exists a maximal separable extension $D$ of $K$ in $L$ such that some relative $p$-basis for $D$ over $K$ remains $p$-independent in $L$.

(4) There exists a proper intermediate field $D$ of $L / K$ such that $L=D\left(K^{p^{-\infty}}\right)$.

Proof. Assume $L=D \bigotimes_{K} K^{p^{-\infty}}$. Then $D$ satisfies properties (2), (3), and (4). Assume (2). Since $D$ is a maximal separable extension of $K$ in $L, L / D$ is purely inseparable. Since $D\left(\left(D\left(K^{p^{-\infty}}\right)\right)^{p}\right)=D\left(K^{p^{-\infty}}\right)$, $D\left(K^{p^{-\infty}}\right)$ is pure in $L / D$ [12, Definition, p. 41]. We claim $D^{p^{-1}} \cap L=$ $D^{p^{-1}} \cap\left(D\left(K^{p^{-\infty}}\right)\right)$. Let $b \in\left(D^{p^{-1}} \cap L\right) \backslash D$. Then $D(b)$ and $K^{p^{-1}}$ are not linearly disjoint over $K$ since $D(b)$ is not separable over $K$. Since $\left[K^{p^{-1}}: K\right]=p, K^{p^{-1}} \subseteq D(b)$ and hence $D(b)=D\left(K^{p^{-1}}\right)$. Thus $b \in D\left(K^{p^{-\infty}}\right)$ and the claim is established. By [12, Proposition 2.7, p. 44], $D\left(K^{p^{-\infty}}\right)=$ $D \otimes_{K} K^{p^{-\infty}}=L$ and (1) holds. Assume (3). A relative $p$-basis $B$ for $D$ over $K$ is a $p$-basis for $D\left(K^{p^{-\infty}}\right)$. Since this $p$-basis remains $p$ $p$-independent in $L, L / D\left(K^{p^{-\infty}}\right)$ is separable. Since $L / D$, whence $L / D\left(K^{p^{-\infty}}\right)$ is also purely inseparable, $L=D \otimes_{K} K^{p^{-\infty}}$ and (1) holds. Assume (4). Since $D$ is proper, $D \nsupseteq K^{p^{-\infty}}$ and hence $D / K$ splits, say $D=D^{\prime} \bigotimes_{K} K^{p^{-n}}$. Then $L=D^{\prime} \bigotimes_{K} K^{p^{-\infty}}$.

II. In this section we determine necessary and/or sufficient conditions for the following to hold for an arbitrary extension $L / K$;

(a) $L^{\prime} / K$ splits for any intermediate field $L^{\prime}$;

(b) $L^{\prime} / K$ is modular for any intermediate field $L^{\prime}$.

We will need the following result.

LEMma 2.1. Suppose $L / K$ splits and the intermediate fields of $\left(L \cap K^{p^{-\infty}}\right) / K$ appear in a chain. If $L^{\prime}$ is an intermediate field of $L / K$, then $L^{\prime} /\left(L^{\prime} \cap K^{p^{-\infty}}\right)$ is separable.

Proof. We first note that $L \cap K^{p-\infty}$ and $L^{\prime}$ are linearly disjoint over $L^{\prime} \cap K^{p^{-\infty}}$. This follows since $L \cap K^{p^{-\infty}} / L^{\prime} \cap K^{p^{-\infty}}$ is purely inseparable and the intermediate fields are chained. Now since $L / K$ splits, $L / L \cap K^{p^{-\infty}}$ is separable and hence $\left(L \cap K^{p^{-\infty}}\right)\left(L^{\prime}\right)$ is separable over $L \cap K^{p^{-\infty}}$. By [6, Corollary 6, p. 266], we conclude $L^{\prime} / K^{p^{-\infty}} \cap$ $L^{\prime}$ is separable.

THEOREM 2.2. Suppose $L / K$ is inseparable but not purely inseparable. Then each condition in the following list implies the succeeding one. 
(1) $L / K$ splits and $\left(L \cap K^{p^{-\infty}}\right) / K$ is simple.

(2) $L^{\prime} / K$ splits for every intermediate field $L^{\prime}$.

(3) $L / K$ splits and the intermediate fields of $\left(L \cap K^{p^{-\infty}}\right) / K$ appear in a chain.

Proof. (1) implies (2): Let $L^{\prime}$ be an intermediate field. By 2.1, $L^{\prime} /\left(L^{\prime} \cap K^{p^{-\infty}}\right)$ is separable. Since $\left(L \cap K^{p^{-\infty}}\right) / K$ is simple, $\left(L^{\prime} \cap K^{p^{-\infty}}\right) / K$ is of bounded exponent and so $L^{\prime} / K$ splits [5, Theorem 4, p. 1178].

(2) implies (3): Suppose the intermediate fields of $\left(L \cap K^{p^{-\infty}}\right) / K$ do not appear in a chain. Then there exist $b, c$ in $L \cap K^{p^{-\infty}}$ such that $b \notin K(c), c \notin K(b)$, and both $b$ and $c$ have some positive exponent $i$ over $K$. Let $z \in L \backslash K$ be such that $K(z) / K$ is separable. Let $L^{\prime}=$ $K(z, z b+c)$. Now $K(z)$ is a distinguish maximal separable intermediate field of $L^{\prime} / K$. Since $L^{\prime} / K$ splits, $L^{\prime}=K(z) \bigotimes_{K} J$ by [8, Lemma, p. 607] where $J=L^{\prime} \cap K^{p^{-\infty}}$. Let $\mathscr{Z}$ be a linear basis of $K(z) / K$ with $1, z \in \mathbb{X}$. Now $z b+c=\sum x_{j} d_{j}$ where $x_{j} \in \mathscr{X}$ and $d_{j} \in J$. Thus

$$
z^{p^{i}} b^{p^{i}}+c^{p^{i}}=\sum x_{j}^{p^{i}} d_{j}^{p^{i}}, d_{j}^{p^{i}} \in J^{p^{i}} \cap K .
$$

Since $K(z) / K$ is separable, $z^{p^{2}}$ is linearly independent over $K$. Hence by equating coefficients in $\left(^{*}\right)$ we have $b^{p^{i}}, c^{p^{i}} \in J^{p^{i}}$. Thus $b, c \in J$. Hence $[J: K]>p^{2}$ and $\left[L^{\prime}: K(z)\right]>p^{i}$, a contradiction. Hence the intermediate fields of $\left(L \cap K^{p^{-\infty}}\right) / K$ appear in a chain.

Corollary 2.3. Suppose $L / K$ has finite inseparability exponent. Then the following conditions are equivalent.

(1) $L^{\prime} / K$ is modular for every intermediate field $L^{\prime}$.

(2) $L / K$ is modular and $\left(L \cap K^{p^{-\infty}}\right) / K$ is simple.

(3) $L^{\prime} / K$ splits for every intermediate field $L^{\prime}$.

(4) $L / K$ splits and $\left(L \cap K^{p^{-\infty}}\right) / K$ is simple.

Proof. A modular field extension with finite inseparability exponent splits. Also a field extension which splits and whose maximal purely inseparable subfield is simple is necessarily modular.

Corollary 2.4. Suppose $L / K$ is inseparable and $L /\left(L \cap K^{p^{-\infty}}\right)$ has a finite separating transcendence basis. Then the following conditions are equivalent.

(1) $L^{\prime} / K$ is modular for every intermediate field $L^{\prime}$.

(2) $L^{\prime} / K$ splits for every intermediate field $L^{\prime}$.

(3) The intermediate fields of $\left(L \cap K^{p^{-\infty}}\right) / K$ appear in a chain.

Proof. Assume (1). Let $L^{\prime}$ be an intermediate field of $L / K$. If $L^{\prime} \supseteqq L \cap K^{p^{-\infty}}$, then $L^{\prime} / L \cap K^{p^{-\infty}}$ has a finite separating transcendence 
basis [10, Theorem 1, p. 418] so $L^{\prime} / K$ splits by [4, Proposition $1, \mathrm{p}$. 2]. If $L^{\prime} \nsupseteq L \cap K^{p^{-\infty}}$, then $L^{\prime} \cap K^{p^{-\infty}} / K$ is a simple extension since the intermediate fields of $L \cap K^{p^{-\infty}} / K$ must be chained. For if they are not chained, choose $b, c, z$ as in the proof of 2.2 and $K(z, z b+c)$ is not modular over $K$. Now we have $\left(L^{\prime} \cap K^{\left.p^{-\infty}\right) / K}\right.$ simple of bounded exponent, $L^{\prime} / L^{\prime} \cap K^{p^{-\infty}}$ separable since $L^{\prime} / K$ is assumed modular, and hence $L^{\prime} / K$ splits by [5, Theorem 4, p. 1178].

That (2) implies (3) is part of 2.2. Assume (3). Since $L / L \cap K^{p^{-\infty}}$ has a finite separating transcendence basis, $L / K$ splits. By 2.1 , if $L^{\prime}$ is an intermediate field of $L / K$, then $L^{\prime} / L^{\prime} \cap K^{p^{-\infty}}$ is separable. Since $L^{\prime} \cap K^{p^{-\infty}} / K$ is modular, $L^{\prime} / K$ is modular by [11, Lemma 5(3), p. 407].

Corollary 2.5. Suppose $L / K$ is inseparable but not purely inseparable. Then each statement in the following list implies the succeeding one.

(1) $L / K$ is modular and $\left(L \cap K^{p^{-\infty}}\right) / K$ is simple.

(2) $L^{\prime} / K$ is modular for every intermediate field $L^{\prime}$.

(3) $L / K$ is modular and the intermediate fields of $\left(L \cap K^{p^{-\infty}}\right) / K$ appear in a chain.

Proof. Straightforward.

III. We now determine necessary and sufficient conditions for the following to hold;

(a) $L / L^{\prime}$ splits for any intermediate field $L^{\prime}$.

(b) $L / L^{\prime}$ is modular for any intermediate field $L^{\prime}$.

THEOREM 3.1. (1) Suppose $L \nsupseteq K^{p^{-\infty}}$. Then $L / L^{\prime}$ splits for every intermediate field $L^{\prime}$ of $L / K$ if and only if $L / K$ is algebraic and $L / K$ splits.

(2) Suppose $L \supseteqq K^{p^{-\infty}}$. Then $L / L^{\prime}$ splits for every intermediate field $L^{\prime}$ of $L / K$ if and only if $L=L^{p}$.

Proof. (1) Suppose $L / L^{\prime}$ splits for every intermediate field $L^{\prime}$. Let $J=L \cap K^{p^{-\infty}}$ and let $b \in J \backslash J^{p}$. Theh $b^{p^{-1}} \notin L$. Assume $L / J$ is not algebraic and let $z \in T$, where $T$ is a transcendence basis for $L / J$. Let $\hat{J}=J(T \backslash\{z\})$ :and $w=-z^{2 p}\left(z^{p}+b\right)^{-1}$. Then $z^{2 p}+w z^{p}+w b=0$. The polynomial $X^{2 p}+w X^{p}+w b$ is irreducible over $\hat{J}(w)$ by Eisenstein's criterion. However $L / \widehat{J}(w)$ does not split else $w^{p^{-1}}, w^{p^{-1}} b^{p^{-1}} \in L$ by [9, Lemma 3.7, p. 102] and so $b^{p^{-1}} \in L$. Thus $L / K$ is algebraic. Clearly $L / K$ splits. Conversely, suppose $L / K$ is algebraic and splits, say $L=S \bigotimes_{K} J$ where $S / K$ is separable algebraic. Then $L=$ 
$S L^{\prime} \bigotimes_{L^{\prime}} J L^{\prime}$ for every intermediate field $L^{\prime}$ of $L / K$.

(2) Suppose $L / L^{\prime}$ splits for every $L^{\prime}$. If $L / K$ is algebraic, then $L$ is separable algebraic over the perfect field $K^{p^{-\infty}}$, and is thus perfect. Suppose $L / K$ is not algebraic and $L \neq L^{p}$. Let $b \in L \backslash L^{p}$. Then $L / \hat{J}(w)$ does not split, a contradiction, where $\hat{J}(w)$ is the field defined in (1) above. Hence $L=L^{p}$. Conversely, suppose $L=L^{p}$. Let $L^{\prime}$ be an intermediate field of $L / K$. Since $L=L^{p}, L^{\prime p^{-\infty}} \cong L$. Hence $L / L^{\prime}$ splits by 1.3 .

THEOREM 3.2. (1) Suppose $L \nsupseteq K^{p^{-\infty}}$. Then $L / L^{\prime}$ is modular for every intermediate field $L^{\prime}$ of $L / K$ if and only if $L / K$ is algebraic splits and $L / L^{\prime}$ is modular for every intermediate field $L^{\prime}$ of $L / S$ where $S$ is the maximal separable intermediate field of $L / K$.

(2) Suppose $L \supseteqq K^{p^{-\infty}}$. Then $L / L^{\prime}$ is modular for every intermediate field $L^{\prime}$ of $L / K$ if and only if $L=L^{p}$.

Proof. (1) Suppose $L / L^{\prime}$ is modular for every $L^{\prime}$. If $L / K$ is not algebraic, then we can construct the field $\hat{J}(w)$ of 3.1. Since $L / \widehat{J}(w)$ is algebraic, $L / \hat{J}(w)$ cannot be modular else it would split. Thus $L / K$ is algebraic. Since $L / K$ is also modular, $L / K$ splits. Conversely, suppose $L / K$ is algebraic and splits and $L / L^{\prime}$ is modular for every intermediate field $L^{\prime}$ of $L / S$. Then $L / L^{\prime}$ is modular for every intermediate field $L^{\prime}$ of $L / K$ by [7, Lemma 4, p. 340] since $L / L^{\prime}$ necessarily splits.

(2) Suppose $L / L^{\prime}$ is modular for every $L^{\prime}$. If $L / K$ is algebraic, then $L$ is separable algebraic over the perfect field $K^{p^{-\infty}}$ and is thus perfect. Suppose $L / K$ is not algebraic and $L \neq L^{p}$. Let $b \in L \backslash L^{p}$. Then $L / \hat{J}(w)$ is not modular, a contradiction, where $\hat{J}(w)$ is the field defined in 3.1. Hence $L=L^{p}$. Conversely, suppose $L=$ $L^{p}$. Let $L^{\prime}$ be an intermediate of $L / K$. By 3.1(2), $L / L^{\prime}$ splits, say $L=D \otimes_{L^{\prime}} L^{p^{-\infty}}$ where $D$ is separable over $L^{\prime}$. Since $L^{p^{-\infty}} / L^{\prime}$ is modular, $L / L^{\prime}$ is modular.

CoROLlaRy 3.3. Let $L$ be a perfect field. Then $L$ splits over every subfield.

Proof. $Z_{p}^{p^{-\infty}}=Z_{p} \subseteq L$ and $L=L^{p}$.

COROLlaRy 3.4. Consider the following statements:

(a) $L / L^{\prime}$ and $L^{\prime} / K$ split for every intermediate field $L^{\prime}$.

(b) $L / L^{\prime}$ and $L^{\prime} / K$ are modular for every intermediate field $L^{\prime}$.

(c) $L / K$ is algebraic and splits and the intermediate fields of $\left(L \cap K^{p^{-\infty}}\right) / K$ appear in chain.

(d) $L=L^{p}$ and $\left[K: K^{p}\right] \leqq p$. 


\section{Then; (1) Suppose $L \nsupseteq K^{p^{-\infty}}$. Then $(\mathrm{a}) \Leftrightarrow(\mathrm{b}) \Leftrightarrow(\mathrm{c})$. \\ (2) Suppose $L \supseteq K^{p^{-\infty}}$. Then $(\mathrm{a}) \Rightarrow(\mathrm{b}) \Leftrightarrow(\mathrm{d})$.}

\section{Proof. Straightforward.}

\section{REFERENCES}

1. J. Deveney, A counterexample concerning inseparable field extensions, Proc. Amer. Math. Soc., 55 (1976), 33-34.

2. J. Deveney and J. Mordeson, Subfields and invariants of inseparable field extensions, preprint.

3. J. Dieudonne, Sur les extensions transcendantes separables, Summa Brasil. Math., 2 (1947), 1-20.

4. N. Heerema and D. Tucker, Modular field extensions, Proc. Amer. Math. Soc., 53 (1975), 1-6.

5. H. Kreimer and N. Heerema, Modularity vs. separability for field extensions, Canad.

J. Math., 27 (1975), 1176-1182.

6. S. Lang, Algebra, Addison-Wesley, Reading, Mass., 1967.

7. J. Mordeson, On a Galois theory for inseparable field extensions, Trans. Amer. Math. Soc., 214 (1975), 337-347.

8. - Splitting of field extensions, Arch. Math., 26 (1975), 606-610.

9. J. Mordeson and B. Vinograde, Structure of arbitrary purely inseparable field extensions, Lecture Notes in Math., Vol. 73, Springer-Verlag, Berlin and New York (1970). 10. - Separating p-bases and transcendental field extensions, Proc. Amer, Math. Soc., 31 (1972), 417-422.

11. M. E. Sweedler, Structure of inseparable extensions, Ann. of Math., (2) 87 (1968), 401-410.

12. W. Waterhouse, The structure of inseparable field extensions, Trans. Amer. Math. Soc., 211 (1975), 39-56.

13. M. Weisfeld, Purely inseparable extensions and higher derivations, Trans. Amer. Math. Soc., 116 (1965), 435-449.

Received October 27, 1973. Supported by the Grants-in-Aid Program for Faculty of Virginia Commonwealth University.

Virginia Commonwealth UNIVESRITY

RICHMOND, VA 23284

AND

CREIGHTON UNIVERSITY

ОмAнA, NB 68131 


\section{PACIFIC JOURNAL OF MATHEMATICS}

EDITORS

DONALD BABBITT (Managing Editor)

University of California

Los Angeles, California 90024

Hugo RossI

University of Utah

Salt Lake City, UT 84112

C. C. MOORE and ANDREW OGG

University of California

Berkeley, CA 94720

\section{J. DUGUNDJI}

Department of Mathematics University of Southern California Los Angeles, California 90007

R. Finn and J. Milgram Stanford University

Stanford, California 94305

\section{ASSOCIATE EDITORS}
E. F. BECKENBACH
B. H. Neumann
F. WOLF
K. YoSHIDA

\section{SUPPORTING INSTITUTIONS}

UNIVERSITY OF BRITISH COLUMBIA

CALIFORNIA INSTITUTE OF TECHNOLOGY

UNIVERSITY OF CALIFORNIA

MONTANA STATE UNIVERSITY

UNIVERSITY OF NEVADA, RENO

NEW MEXICO STATE UNIVERSITY

OREGON STATE UNIVERSITY

UNIVERSITY OF OREGON
UNIVERSITY OF SOUTHERN CALIFORNIA

STANFORD UNIVERSITY

UNIVERSITY OF HAWAII

UNIVERSITY OF TOKYO

UNIVERSITY OF UTAH

WASHINGTON STATE UNIVERSITY

UNIVERSITY OF WASHINGTON 


\section{Pacific Journal of Mathematics}

\section{Vol. 83, No. 1 \\ March, 1979}

Richard Neal Ball, Topological lattice-ordered groups ............... 1

Stephen Berman, On the low-dimensional cohomology of some

infinite-dimensional simple Lie algebras .................. 27

R. P. Boas and Gerald Thomas Cargo, Level sets of derivatives ......... 37

James K. Deveney and John Nelson Mordeson, Splitting and modularly

perfect fields......................................

Robert Hugh Gilman and Ronald Mark Solomon, Finite groups with small

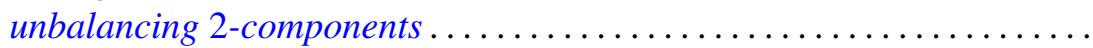

George Grätzer, Andras Hajnal and David C. Kelly, Chain conditions in free products of lattices with infinitary operations..................

Benjamin Rigler Halpern, Periodic points on tori ..................

Dean G. Hoffman and David Anthony Klarner, Sets of integers closed under

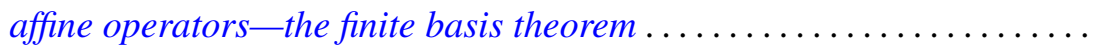

Rudolf-Eberhard Hoffmann, On the sobrification remainder ${ }^{s} X-X \ldots \ldots$

Gerald William Johnson and David Lee Skoug, Scale-invariant

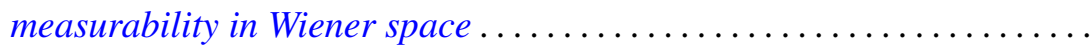

Michael Keisler, Integral representation for elements of the dual of

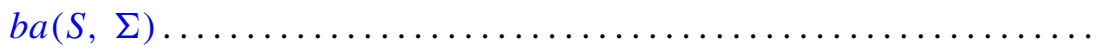

Wayne C. Bell and Michael Keisler, A characterization of the representable Lebesgue decomposition projections ................

Wadi Mahfoud, Comparison theorems for delay differential equations ...

R. Daniel Mauldin, The set of continuous nowhere differentiable functions .

Robert Wilmer Miller and Mark Lawrence Teply, The descending chain condition relative to a torsion theory...

Yoshiomi Nakagami and Colin Eric Sutherland, Takesaki's duality for regular extensions of von Neumann algebras ........ .

William Otis Nowell, Tubular neighborhoods of Hilbert cube manifolds ...

Mohan S. Putcha, Generalization of Lentin's theory of principal solutions of word equations in free semigroups to free product of copies of positive reals under addition

Amitai Regev, A primeness property for central polynomials . ...

Saburou Saitoh, The Rudin kernels on an arbitrary domain. . .

Heinrich Steinlein, Some abstract generalizations of the

Ljusternik-Schnirelmann-Borsuk covering theorem . . . 